\title{
Modeling of the three-dimensional motion of toroidal magnetic clouds in the inner heliosphere
}

\author{
E. Romashets ${ }^{1,2,3}$, M. Vandas ${ }^{4}$, and S. Poedts ${ }^{1}$ \\ 1 Center for Plasma Astrophysics, K.U.Leuven, 3001 Leuven, Belgium \\ e-mail: Stefaan.Poedts@wis.kuleuven.be \\ 2 IZMIRAN, Troitsk, Moscow Region 142190, Russia \\ 3 Solar Observatory of Prairie View A\& M University, Prairie View 77446, USA \\ 4 Astronomical Institute, Boční II 1401, 14131 Praha 4, Czech Republic
}

Received 9 August 2006 / Accepted 19 January 2007

ABSTRACT

\begin{abstract}
Context. The motion of a magnetic cloud through the heliosphere is governed by three main forces, viz. the diamagnetic force, the drag force, and gravity. Some recently derived formulas enabling the calculation of the ambient magnetic field around a toroidal magnetic cloud are applied to calculate the diamagnetic force acting on the cloud and to determine the cloud dynamics.

Aims. The aim is to determine the three dimensional velocity profiles and the trajectory of the magnetic cloud, as well as the evolution of the orientation of the cloud axis from the calculated moment of the force.

Methods. The method applied in this study consists of three steps. First, the $r$-component of the magnetic field at $r=2.5 R_{\mathrm{s}}$ is derived from a spherical harmonic analysis. Next, the field distribution in the entire heliosphere, including the spiral structure, is reconstructed in a way that is consistent with this boundary condition at $r=2.5 R_{\mathrm{s}}$ as well as with actual measurements at 1 AU. Then, a toroid is launched at a point obtained from solar observations of a specific event and the initial size, orientation, and velocity of this toroid is estimated from these observational data as well.

Results. The three dimensional velocity profiles and the trajectory of the magnetic cloud, as well as the evolution of the orientation of the cloud axis have been determined for a toroidally shaped cloud moving in the interplanetary medium taking into account a spiral magnetic field.
\end{abstract}

Key words. Sun: coronal mass ejections (CMEs) - Sun: solar wind - Sun: magnetic field - Sun: solar-terrestrial relations

\section{Introduction and motivation}

Coronal Mass Ejections (CMEs) play a crucial role in space weather and a careful study of the origin, the structure, and the propagation characteristics of this violent phenomenon is essential for a deeper insight into space weather physics. The fast CMEs are important because of the geomagnetic storms created by the impact of the CMEs on the Earth's magnetosphere and the solar energetic particles (SEPs) accelerated by the CME leading shock front. Therefore, it is important to learn more about the dynamics of the most geo-effective coronal mass ejections (CMEs) in order to be able to predict their geo-effectiveness, i.e. how much geomagnetic activity they will trigger upon their arrival in the Earth's vicinity and their interaction with the magnetosphere.

Until recently, there was only one tool to monitor the CME velocity, viz. radio observations (Cane et al. 1986; Reiner et al. 2005). In fact, from these radio data one can first determine the location of the bow shock ahead of a fast CME, and then calculate the location of the CME or magnetic cloud itself on the basis of some mathematical models or assumptions. The new techniques for interpretation of SOHO LASCO images (Sheeley et al. 1999) and of Solar Mass Ejection Imager (SMEI) data (Howard et al. 2006), open possibilities to detect the CME velocity more precisely and, thus, to improve the predictions of their time of arrival.

To construct a reliable analytical or numerical MHD model for the calculation of such velocity profiles, one should take into account the following circumstances: the interplanetary medium through which the CME is traveling has a very complicated structure that is, in addition, evolving rapidly with time. The interaction between high speed and low speed flows in the solar wind is one of the most important sources of inhomogeneity there. As a result, the heliospheric current sheet (HCS) and the heliospheric plasma sheet (HPS) do not have an ideal spiral structure (Winterhalter et al. 1994). On the other hand, these surfaces can compress the CMEs and increase their geo-efficiency (Ivanov et al. 1992; Ivanov et al. 1996). The dynamics of toroidal magnetic clouds was considered in detail by Romashets \& Vandas (2001) and Vandas et al. (2003). Vandas et al. (2003) also derived formulas for the moments of the diamagnetic force. In these papers, however, a simplifying approach has been followed by assuming an isolated toroidal magnetic cloud that is moving along a straight line through a spherically symmetric heliosphere. In the present paper, we propose a much more realistic model in which the considered cloud moves in a structured interplanetary medium with a spiral magnetic field.

In the next section we present the mathematical and physical model including the equation of motion that is solved and the derivation of the diamagnetic force acting on the magnetic cloud (with constant or variable aspect ratio) and the assumption made for the expansion of the magnetic cloud and the reconstruction of the IMF spiral structure, consistent with the source-surface magnetic field. In Sect. 3 we apply the results in order to demonstrate 


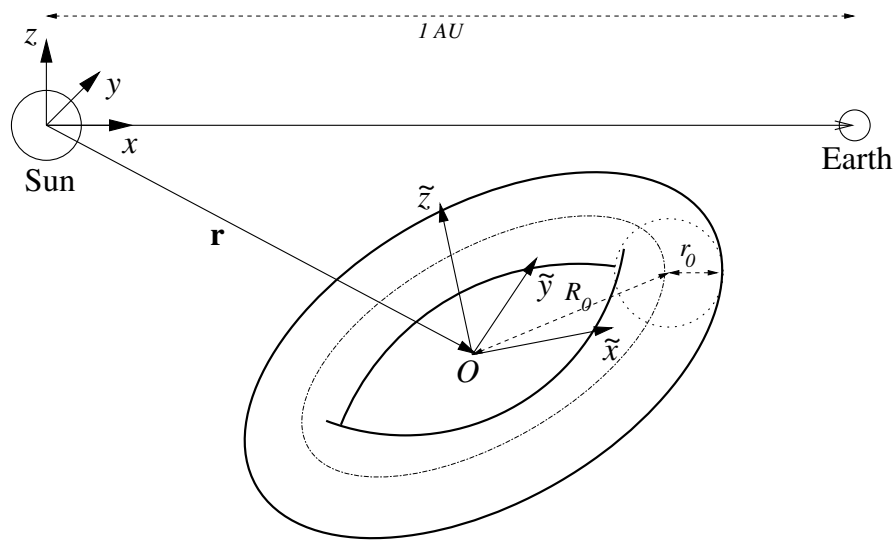

Fig. 1. Sketch of the relation between the solar ecliptic coordinate system (SEC) and the chosen local coordinate system (sizes of Earth and Sun are not to the scale).

the strength of the model. The results are discussed in Sect. 4, followed by our conclusions in Sect. 5 .

\section{Mathematical and physical model}

\subsection{The equation of motion}

Consider a magnetic cloud that has the shape of a torus. Assume that the axis of symmetry of the toroid is oriented along the unit vector $\boldsymbol{a}=\left(a_{x}, a_{y}, a_{z}\right)$ in the solar ecliptic coordinate system (SEC). Consider a local coordinate system that has its center at the symmetry point of the toroid, $\boldsymbol{r}\left(r_{x}, r_{y}, r_{z}\right)$, its $\tilde{z}$-axis along $\boldsymbol{a}$, and the $\tilde{x}$-axis in the direction normal to the $\tilde{z}$-axis and in the plane described by the vectors $\boldsymbol{r}$ and $\boldsymbol{a}$. The $\tilde{y}$-axis then simply completes the right handed vector basis. The geometrical situation is shown schematically in Fig. 1.

We thus have

$$
\begin{aligned}
& \boldsymbol{e}_{\tilde{x}}=\frac{(\boldsymbol{a} \times \boldsymbol{r}) \times \boldsymbol{a}}{\|(\boldsymbol{a} \times \boldsymbol{r}) \times \boldsymbol{a}\|}, \\
& \boldsymbol{e}_{\tilde{y}}=\frac{\boldsymbol{a} \times \boldsymbol{r}}{\|\boldsymbol{a} \times \boldsymbol{r}\|}, \\
& \boldsymbol{e}_{\tilde{z}}=\boldsymbol{a} .
\end{aligned}
$$

Since the diamagnetic force has all three components, we end up with a three-dimensional equation of motion:

$$
\ddot{\boldsymbol{r}}=\dot{\boldsymbol{V}}=\boldsymbol{F} / M_{\mathrm{c}} \text {. }
$$

Here, $M_{\mathrm{c}}$ corresponds to the total mass of the magnetic cloud while $\boldsymbol{F}$ denotes the vector sum of all three forces acting on the toroidal body, viz. the diamagnetic force, gravity, and the drag force. Numerically integrating Eq. (4), e.g. by a simple forward Euler scheme, we obtain:

$$
\boldsymbol{V}^{k+1}=\boldsymbol{F} \Delta t / M_{\mathrm{c}}+\boldsymbol{V}^{k},
$$$$
\boldsymbol{r}^{k+1}=\boldsymbol{V}^{k+1} \Delta t+\boldsymbol{r}^{k} \text {. }
$$

The drag force $F_{\mathrm{d}}$ is given by

$F_{\mathrm{d}}= \pm K N_{\mathrm{e}} m_{\mathrm{p}}\left(V_{\mathrm{sw}}-V\right)^{2} S$.

The Newtonian constant $K$ is set to unity, $m_{\mathrm{p}}$ denotes the proton mass, and $N$ and $V_{\text {sw }}$ correspond to the number density and the velocity of the background solar wind, respectively. The toroid's cross section $S$ is variable and is approximately equal to

$$
S \simeq \pi r_{0}^{2}\left(1-\cos ^{2} \beta_{1}\right)+4 R_{0} r_{0}\left[1+(\pi-1) \cos ^{2} \beta_{1}\right] .
$$

Here, $r_{0}$ denotes the toroid's small radius while $R_{0}$ corresponds to the large radius of the toroid (see Fig. 1). The angle between $\boldsymbol{a}$ and $\boldsymbol{r}$ is indicated by $\beta_{1}$. The gravitational force $F_{\mathrm{g}}$ is given by

$F_{\mathrm{g}}=-\frac{G M_{\mathrm{S}} M_{\mathrm{c}}}{r^{2}}$

where $G$ is the gravitational constant, $r$ is the helio-distance, i.e. the distance between the center of the Sun and the center of the toroid (in other words, $r=\|\boldsymbol{r}\|$ ), and $M_{\mathrm{S}}$ denotes the solar mass.

\subsection{The diamagnetic force acting on a magnetic cloud with constant aspect ratio}

To calculate the diamagnetic force, $\boldsymbol{F}_{\mathrm{dm}}$, considered earlier in Parker (1957) and in Pneuman (1984), we should first determine the modification of the ambient magnetic field caused by the insertion of the magnetic cloud. We can write a linear approximation of the real ambient field in the vicinity of the point $\mathrm{O}$ (see Fig. 1) in the form:

$$
\begin{aligned}
B_{x}= & B_{0 x}+\frac{B_{1 x x}}{L}\left(x-r_{x}\right)+\frac{B_{1 x y}}{L}\left(y-r_{y}\right)+\frac{B_{1 x z}}{L}\left(z-r_{z}\right) \\
B_{y}= & B_{0 y}+\frac{B_{1 x y}}{L}\left(x-r_{x}\right)+\frac{B_{1 y y}}{L}\left(y-r_{y}\right)+\frac{B_{1 y z}}{L}\left(z-r_{z}\right) \\
B_{z}= & B_{0 z}+\frac{B_{1 x z}}{L}\left(x-r_{x}\right)+\frac{B_{1 y z}}{L}\left(y-r_{y}\right) \\
& -\frac{B_{1 x x}+B_{1 y y}}{L}\left(z-r_{z}\right)
\end{aligned}
$$

This linear field is given by the potential

$$
\begin{aligned}
\Phi_{0}= & B_{0 x}\left(x-r_{x}\right)+B_{0 y}\left(y-r_{y}\right)+B_{0 z}\left(z-r_{z}\right) \\
& +\frac{B_{1 x x}}{2 L}\left(x-r_{x}\right)^{2}+\frac{B_{1 y y}}{2 L}\left(y-r_{y}\right)^{2} \\
& -\left(\frac{B_{1 x x}+B_{1 y y}}{2 L}\right)\left(z-r_{z}\right)^{2}+\frac{B_{1 x y}}{L}\left(x-r_{x}\right)\left(y-r_{y}\right) \\
& +\frac{B_{1 y z}}{L}\left(y-r_{y}\right)\left(z-r_{z}\right)+\frac{B_{1 x z}}{L}\left(x-r_{x}\right)\left(z-r_{z}\right) .
\end{aligned}
$$

Here $B_{0 x x} / L=\partial B_{x} / \partial x$, etc.; $B_{0 x y} / L=\left(\partial B_{x} / \partial y+\partial B_{y} / \partial x\right) / 2$, etc. (in the point $\mathrm{O}$ ). Hence, the real ambient field is fitted by a linear potential field at any point.

The relation between distances in the SEC and the coordinates (1)-(3) is given, in terms of Euler angles $\alpha$ and $\beta$, by

$x=r_{x}+\tilde{x} \sin \alpha+\tilde{z} \cos \alpha$,

$y=r_{y}-\tilde{x} \cos \alpha \cos \beta+\tilde{y} \sin \beta+\tilde{z} \sin \alpha \cos \beta$,

$z=r_{z}-\tilde{x} \cos \alpha \sin \beta-\tilde{y} \cos \beta+\tilde{z} \sin \alpha \sin \beta$.

The scalar magnetic potential (11) is then expressed in the intrinsic (tilde) system through the substitution by Eqs. (12)-(14), symbolically as

$\Phi_{0}=\sum_{i=1}^{8} C_{i} \Phi_{0}^{i}$,

i.e., it is arranged as a sum of the following harmonic functions, $\Phi_{0}^{1}=\tilde{x}, \Phi_{0}^{2}=\tilde{y}, \Phi_{0}^{3}=\tilde{z}, \Phi_{0}^{4}=\tilde{x}^{2}-\tilde{y}^{2}$,

$\Phi_{0}^{5}=\frac{\tilde{x}^{2}+\tilde{y}^{2}}{2}-\tilde{z}^{2}, \Phi_{0}^{6}=\tilde{x} \tilde{y}, \Phi_{0}^{7}=\tilde{x} \tilde{z}, \Phi_{0}^{8}=\tilde{y} \tilde{z}$.

The coefficients $C_{i}$ in Eq. (15) are given in Appendix A. 
Now we shall look for the perturbed potential magnetic field (due to the presence of the toroidal cloud) for which the normal components $B_{\mathrm{n}}$ to the surface of the flux rope are zero (condition 1) and that approaches the undisturbed field (10) at large distances from the flux rope (condition 2 ). That is, we add to the scalar potentials $\Phi_{0}^{i}$ in Eq. (16) additional harmonic functions, for which the additional magnetic field goes to zero for large distances from the rope and for which the total magnetic field components $B_{\mathrm{n}}^{i}=0$ at the flux rope boundary. This is done formally by transitions $\Phi_{0}^{i} \rightarrow \Phi^{i}$, where the $\Phi^{i}$ are harmonic functions fulfilling the above-mentioned conditions 1 and 2 . The resulting scalar magnetic potential $\Phi$ reads

$\Phi=\sum_{i=1}^{8} C_{i} \Phi^{i}$

and the magnetic field components directly follow from

$\boldsymbol{B}=\operatorname{grad} \Phi$.

The intrinsic system of the toroid will be expressed in the toroidal coordinates $\mu, \eta$, and $\varphi$ :

$\tilde{x}=\frac{a \sinh \mu \cos \varphi}{\cosh \mu-\cos \eta}$,

$\tilde{y}=\frac{a \sinh \mu \sin \varphi}{\cosh \mu-\cos \eta}$

$\tilde{z}=\frac{a \sin \eta}{\cosh \mu-\cos \eta}$.

The parameter $a$ equals $\sqrt{R_{0}^{2}-r_{0}^{2}}$, and the surface of the toroid is given by $\mu=\mu_{0}$, where $\cosh \mu_{0}=R_{0} / r_{0}$. A more detailed description of this toroidal coordinate system with an instructive figure can be found in Romashets \& Vandas (2001).

The harmonic functions $\Phi_{0}^{i}$ are given in Appendix B.

To meet the conditions 1 and 2, additional toroidal harmonic functions will be added to the scalar potentials $\Phi_{0}^{i}$ (given by Eqs. (B.1)-(B.8)) which become negligible when $\mu \rightarrow 0$ (i.e. at large distances), and for which the total magnetic field component $B_{\mu}=0$ for $\mu=\mu_{0}$ (i.e. at the surface of the toroid). The resulting harmonics $\Phi^{i}$ are

$$
\begin{aligned}
\Phi^{1}= & \sqrt{\cosh \mu-\cos \eta} \frac{2^{\frac{3}{2}} a}{\pi} \sum_{n=-\infty}^{\infty}\left[Q_{n-\frac{1}{2}}^{1}(\cosh \mu)\right. \\
& \left.-a_{n}^{1} P_{n-\frac{1}{2}}^{1}(\cosh \mu)\right] \cos n \eta \cos \varphi
\end{aligned}
$$

and similarly for the other $\Phi^{i}$, e.g.,

$$
\begin{aligned}
\Phi^{3}= & \sqrt{\cosh \mu-\cos \eta} \frac{2^{\frac{3}{2}} a}{\pi} \sum_{n=-\infty}^{\infty}\left[Q_{n-\frac{1}{2}}(\cosh \mu)\right. \\
& \left.-a_{n}^{3} P_{n-\frac{1}{2}}(\cosh \mu)\right] n \sin n \eta .
\end{aligned}
$$

The $P_{n-\frac{1}{2}}^{m}$ are the Legendre functions of the first kind. The coefficients $a_{n}^{i}$ were formally added and will be selected so that the condition $B_{\mu}=0$ is satisfied at $\mu=\mu_{0}$. Due to the symmetry we set $a_{n}^{i}=a_{-n}^{i}$ for all $i$ and $n$.

We shall introduce the following symbols:

$\Phi^{i}=K_{i} \sqrt{\cosh \mu-\cos \eta} N_{i}(\varphi) \sum_{n=-\infty}^{\infty} G_{n}^{i}(\cosh \mu) H_{n}^{i}(\eta)$.
The coefficients $K_{i}$ and functions $N_{i}, H_{n}^{i}$, and $G_{n}^{i}$ are given in Appendix C.

The magnetic field components then directly follow from Eq. (18):

$$
\begin{aligned}
B_{\mu}= & \frac{\sinh \mu \sqrt{\cosh \mu-\cos \eta}}{2 a} \sum_{i=1}^{8} C_{i} K_{i} N_{i}(\varphi) \\
& \times \sum_{n=-\infty}^{\infty}\left[G_{n}^{i}(\cosh \mu)\right. \\
& \left.+2(\cosh \mu-\cos \eta) G_{n}^{i \prime}(\cosh \mu)\right] H_{n}^{i}(\eta), \\
B_{\eta}= & \frac{\sqrt{\cosh \mu-\cos \eta}}{2 a} \sum_{i=1}^{8} C_{i} K_{i} N_{i}(\varphi) \\
& \times \sum_{n=-\infty}^{\infty} G_{n}^{i}(\cosh \mu)\left[\sin \eta H_{n}^{i}(\eta)\right. \\
& \left.+2(\cosh \mu-\cos \eta) H_{n}^{i \prime}(\eta)\right], \\
B_{\varphi}= & \frac{(\cosh \mu-\cos \eta)^{3 / 2}}{a \sinh \mu} \sum_{i=1}^{8} C_{i} K_{i} N_{i}^{\prime}(\varphi) \\
& \times \sum_{n=-\infty}^{\infty} G_{n}^{i}(\cosh \mu) H_{n}^{i}(\eta) .
\end{aligned}
$$

Here, the primes indicate derivatives w.r.t. the function argument. Condition 2 is fulfilled by the fact that we used the Legendre functions of the first kind (see Romashets \& Vandas 2001). Condition 1 requires to fulfill $B_{\mu}=0$ at $\mu=\mu_{0}$ for all $\eta$ and $\varphi$ in Eq. (23). This is done by selection of the coefficients $a_{n}^{i}$, see Appendix D.

The diamagnetic force acting on the toroid is then given by

$\boldsymbol{F}_{\mathrm{dm}}=-\frac{1}{2 \tilde{\mu}_{0}} \oint\left(B_{\eta}^{2}+B_{\varphi}^{2}\right) \mathrm{d} \boldsymbol{S}$,

because it follows from Eq. (23) that $B_{\mu}=0$ at the flux rope surface (i.e. for $\mu=\mu_{0}$ ). Here, $\tilde{\mu}_{0}$ is the magnetic permeability. We shall calculate the diamagnetic force in the toroid's (tilde) system:

$\boldsymbol{F}_{\mathrm{dm}}=-\frac{1}{2 \tilde{\mu}_{0}} \int_{-\pi}^{\pi} \int_{0}^{2 \pi}\left(B_{\eta}^{2}+B_{\varphi}^{2}\right) \boldsymbol{w} \mathrm{d} \varphi \mathrm{d} \eta$,

where

$w_{\tilde{x}}=\frac{a^{2} \sinh \mu_{0}\left(\cosh \mu_{0} \cos \eta-1\right)}{\left(\cosh \mu_{0}-\cos \eta\right)^{3}} \cos \varphi$,

$w_{\tilde{y}}=\frac{a^{2} \sinh \mu_{0}\left(\cosh \mu_{0} \cos \eta-1\right)}{\left(\cosh \mu_{0}-\cos \eta\right)^{3}} \sin \varphi$,

$w_{\tilde{z}}=\frac{a^{2} \sinh ^{2} \mu_{0} \sin \eta}{\left(\cosh \mu_{0}-\cos \eta\right)^{3}}$.

The integral is calculated at $\mu=\mu_{0}$. Remark that the initial linearized field (10) is not planar as in previous studies. However, it still is current-free. An obvious restriction for the use of this method is that the initial ambient field is assumed to change not very strongly at distances of the order $R_{0}$,

Another approach is to assume that the toroid has a variable minor radius, because conditions are different for its leading and trailing parts (Romashets \& Vandas 2005). Note that when the size of the toroidal cloud is small compared to the length scales at which the interplanetary magnetic field (IMF) changes substantially, both approaches should give almost the same force. 
To calculate the modification of the ambient magnetic and the diamagnetic field acting on a super-sonic cloud, we can use the approach described in Romashets \& Vandas (2005b). In fact, an extra factor is added in the expression for the force in Eq. (26), which is a function of the Mach number $M$. This factor is unity when $M<1$. As long as the toroid is still attached to the Sun, the integration in Eq. (26) is performed only within the limits $\varphi_{0}$ and $\varphi_{1}$, the angular coordinates of two foot points of the toroid on the Sun. The approach also works if the cloud is an highly elongated twisted loop. In that case, the loop is divided into several parts (each part with its own $R_{0}, r_{0}$, orientation in space and location of the center), so that each part is treated as a part of some toroid. The diamagnetic force is the sum of several integrals corresponding to the different parts of the loop.

\subsection{The expansion of the magnetic cloud}

Assume now that the small and large radii of the toroidal magnetic cloud evolve with time in the following manner:

$\dot{r}_{0} \propto r^{-\lambda}, \quad$ and $\quad \dot{R}_{0} \propto r^{-\lambda}$,

where $r$ is the heliodistance of the center and $\lambda$ is some constant, and the magnitude of the IMF

$B \propto r^{-\delta}$,

where $\delta$ is another constant. Assume that the expansion is proportional to the local Alfvén velocity, which would be physically quite natural. This means that

$\dot{r}_{0} \propto \frac{B}{\sqrt{N}} \propto r^{1-\delta}$.

Therefore, we have the following relation between the two constants $\lambda$ and $\delta$ in this case:

$\lambda=\delta-1$.

For $B_{r}$ we have approximately $\delta=2$, for $B_{\phi}$ we have $\delta=1$, so that for $B$ we have $\delta$ between 1 and 2 and, thus, $\lambda$ between 0 and 1.

From Eq. (31) we have

$r_{0}^{\lambda+1} \propto t \propto r$

so that

$r_{0} \propto r^{\frac{1}{\lambda+1}}$.

For the ratio of the density inside to the density outside the cloud we find

$\frac{N_{0}}{N} \propto \frac{r_{0}^{-3}}{r^{-2}} \propto r^{2-\frac{3}{\lambda+1}}$.

We have assumed earlier (Romashets \& Vandas 2001) that the size of the toroid is increasing proportionally to the distance from the Sun, i.e.

$r_{0} \propto r$.

Therefore, from Eq. (36) we find that $\lambda=0$ and

$\frac{N_{0}}{N} \propto r^{-1}$.

The density ratio is thus decreasing as the magnetic cloud is approaching the Earth's orbit. This is not so good as we know from in situ measurements that this density ratio at $1 \mathrm{AU}$ is always below unity and sometimes even below 0.1 .

Therefore, it is suggested in this calculation that

$r_{0} \propto r^{2 / 3}$

Again, from Eq. (36) we now find $\lambda=\frac{1}{2}$ and the density ratio now remains constant. As a result, the density inside the magnetic cloud is always lower than the density outside the magnetic cloud during the full evolution history of the cloud. It is widely accepted that the density of undisturbed solar wind plasma is structured around the heliospheric current sheet. This means that we can add an additional factor in the relationship (40), which is a function of the angular distance from the HCS, say in the form used by Kotova et al. (1987).

\subsection{The IMF spiral structure, consistent with the source-surface field}

Assume that the spherical harmonic analysis gives a $B_{r}^{0}$ component at $r_{\mathrm{ss}}=2.5 R_{\mathrm{s}}$, while the two other components of the magnetic field vanish at this height. The scalar potential is then given by:

$$
\begin{aligned}
\Phi= & B_{0} \sum_{j=0}^{J_{\max }} \sum_{m=0}^{j}\left(\frac{r^{j}}{r_{\mathrm{ss}}^{j-1}}-\frac{r_{\mathrm{ss}}^{j+2}}{r^{j+1}}\right) \\
& \times \sqrt{q_{m} \frac{(j-m) !}{(j+m) !}} P_{j}^{m}(\cos \theta)\left(g_{j}^{m} \cos m \varphi+h_{j}^{m} \sin m \varphi\right),
\end{aligned}
$$

where $q_{m}$ equals 1 for $m=0$, and 2 for $m>0$, since we use the Schmidt normalization of the spherical harmonics. Here, $P_{j}^{m}$ is defined as

$$
\begin{aligned}
P_{j}^{m}(x)= & \frac{\left(1-x^{2}\right)^{m / 2}}{2^{j} j !} \frac{d^{j+m}}{d x^{j+m}}\left(x^{2}-1\right)^{j} \\
= & \frac{1}{2^{m}} \frac{(j+m) !}{(j-m) ! m !}\left(1-x^{2}\right)^{m / 2} \\
& \times F\left(m-j, m+j+1, m+1, \frac{1-x}{2}\right),
\end{aligned}
$$

where $F$ denotes the hypergeometric function.

$F(\tilde{a}, \tilde{b}, \tilde{c}, x)=1+\frac{\tilde{a} \tilde{b}}{\tilde{c}} x+\frac{1}{2 !} \frac{\tilde{a}(\tilde{a}+1) \tilde{b}(\tilde{b}+1)}{\tilde{c}(\tilde{c}+1)} x^{2}+\ldots$

The magnetic field on the source surface is then derived from Eq. (41). It is given by

$$
\begin{aligned}
B_{r}^{0}= & B_{0} \sum_{j, m}(2 j+1) \sqrt{q_{m} \frac{(j-m) !}{(j+m) !}} \\
& \times P_{j}^{m}(\cos \theta)\left(g_{j}^{m} \cos m \varphi+h_{j}^{m} \sin m \varphi\right), \\
B_{\varphi}^{0}= & 0, \quad B_{\theta}^{0}=0 .
\end{aligned}
$$

One can see that Eq. (41) contains rapidly decreasing terms, with $1 / r^{J_{\max }}$, so the field at $r=r_{\mathrm{ss}}$ is much weaker than at $r=R_{\mathrm{s}}$. In other words, there are too many field lines returning back to the Sun, and only a small fraction of the lines reaches the level $r=r_{\mathrm{ss}}$. Another approach, which seems now more adequate, is to treat the region between $R_{\mathrm{s}}$ and $r_{\mathrm{ss}}$ as occupied by a force-free field with $\alpha_{0}=$ const, and given as $\boldsymbol{B}=\sum \operatorname{rot}\left(\boldsymbol{e}_{r} \psi_{j}^{\mathrm{m}}\right)$ 
(Chandrasekhar \& Kendall 1957). The formulas are mostly the same, while the radial dependence is less sharp:

$\psi_{j}^{m} \sim \frac{1}{\sqrt{r}} J_{n-1 / 2}\left(\alpha_{0} r\right) P_{j}^{m}(\cos \theta)\left(g_{j}^{m} \cos m \varphi+h_{j}^{m} \sin m \varphi\right)$.

Here, $\alpha_{0}$ is an averaged parameter over the entire considered volume. It is not known, but we can select it from the condition that the field at the source surface and at $1 \mathrm{AU}$ is reasonable and consistent with in situ measurements. We can use also the harmonic functions (42), but multiplied by an extra factor $D$. This factor is to be chosen from the condition that the averaged field near the Earth's orbit at $\theta=0$ (i.e. in the vicinity of the ecliptic plane) is about $5 n T$.

We propose to use the following formulas for the interval $\left\langle r_{\mathrm{ss}}, r_{\mathrm{E}}\right\rangle$ :

$$
\begin{aligned}
& B_{r}=D B_{r}^{0}\left(\varphi+\varphi_{0}\left(\frac{r-r_{\mathrm{ss}}}{r_{E}-r_{\mathrm{ss}}}\right)^{\gamma}+\omega_{0} t, \theta\right) \frac{r_{\mathrm{ss}}^{2}}{r^{2}}, \\
& B_{\varphi}=-D B_{r}^{0}\left(\varphi+\varphi_{0}\left(\frac{r-r_{\mathrm{ss}}}{r_{\mathrm{E}}-r_{\mathrm{ss}}}\right)^{\gamma}+\omega_{0} t, \theta\right) \sin \theta \\
& \times \frac{r_{\mathrm{ss}}^{2}}{r^{2}} \frac{r-r_{\mathrm{ss}}}{r_{\mathrm{E}}}, \\
& B_{\theta}=-\frac{D}{\sin \theta}\left[\gamma \varphi_{0} \frac{r_{\mathrm{ss}}^{2}}{r} \frac{\left(r-r_{s s}\right)^{\gamma-1}}{\left(r_{\mathrm{E}}-r_{\mathrm{ss}}\right)^{\gamma}}-\frac{r_{\mathrm{ss}}^{2}}{r^{2}} \frac{r-r_{\mathrm{ss}}}{r_{\mathrm{E}}}\right] \\
& \times \int \frac{\partial B_{r}^{0}\left(\varphi+\varphi_{0}\left(\frac{r-r_{\mathrm{ss}}}{r_{\mathrm{E}}-r_{\mathrm{ss}}}\right)^{\gamma}+\omega_{0} t, \theta\right)}{\partial \varphi} \sin \theta \mathrm{d} \theta \\
& =-\frac{D}{\sin \theta}\left[\gamma \varphi_{0} \frac{r_{\mathrm{ss}}^{2}}{r} \frac{\left(r-r_{\mathrm{ss}}\right)^{\gamma-1}}{\left(r_{\mathrm{E}}-r_{\mathrm{ss}}\right)^{\gamma}}-\frac{r_{\mathrm{ss}}^{2}}{r^{2}} \frac{r-r_{\mathrm{ss}}}{r_{\mathrm{E}}}\right] \\
& \times \sum_{j, m}(2 j+1) \sqrt{q_{m} \frac{(j-m) !}{(j+m) !}} W_{j}^{m}(\cos \theta) m \\
& \times\left[-g_{j}^{m} \sin m\left(\varphi+\varphi_{0}\left(\frac{r-r_{\mathrm{ss}}}{r_{\mathrm{E}}-r_{\mathrm{ss}}}\right)^{\gamma}+\omega_{0} t\right)\right. \\
& \left.+h_{j}^{m} \cos m\left(\varphi+\varphi_{0}\left(\frac{r-r_{\mathrm{ss}}}{r_{\mathrm{E}}-r_{\mathrm{ss}}}\right)^{\gamma}+\omega_{0} t\right)\right] \text {. }
\end{aligned}
$$

slightly modified. In Eq. (42) $g_{j}^{m}=g_{j}^{m}(t)$ and $h_{j}^{m}=h_{j}^{m}(t)$. In Eq. (46) the following new term is added:

$$
\begin{aligned}
B_{\theta}^{\mathrm{ns}} & =\frac{D}{\sin \theta} \frac{r_{\mathrm{ss}}^{2}}{r} \frac{1}{V_{\mathrm{sw}}} \sum_{j, m}(2 j+1) \sqrt{q_{m} \frac{(j-m) !}{(j+m) !}} W_{j}^{m}(\cos \theta) m \\
& \times\left[-\dot{g}_{j}^{m}\left(t-r / V_{\mathrm{sw}}\right) \sin m\left(\varphi+\varphi_{0}\left(\frac{r-r_{\mathrm{ss}}}{r_{E}-r_{\mathrm{ss}}}\right)^{\gamma}+\omega_{0} t\right)\right. \\
& \left.+\dot{h}_{j}^{m}\left(t-r / V_{\mathrm{sw}}\right) \cos m\left(\varphi+\varphi_{0}\left(\frac{r-r_{\mathrm{ss}}}{r_{\mathrm{E}}-r_{\mathrm{ss}}}\right)^{\gamma}+\omega_{0} t\right)\right] .
\end{aligned}
$$

\section{Application and results}

We now illustrate the strength of the mathematical model presented in the previous sections. We first apply the results of Sect. 2.5 to reconstruct a model IMF through which the sample toroid will propagate. We considered the following set of coefficients: $g_{1}^{0}=9.2, g_{2}^{0}=-0.92, g_{2}^{1}=0.62, g_{2}^{2}=0.63, g_{3}^{2}=0.03$, $h_{2}^{1}=-0.37, h_{2}^{2}=0.104$. The factor $D B_{0}$ in Eqs. (44)-(46) is taken equal to $3 \times 10^{-6} \mathrm{~T}$ in order to have $B=5 \mathrm{nT}$ at $1 \mathrm{AU}$ in the vicinity of the ecliptic plane, as mentioned above.

In Figs. 2-4 the structure of the interplanetary magnetic field given by Eqs. (44)-(46) is illustrated. In Fig. 2 we plotted the sector boundary of the obtained IMF. Figures 3 and 4 contain contour plots of $B_{r}$ in the equatorial plane and of $B$ in a plane parallel to the equatorial plane, respectively. In Fig. 5 we show $B_{r}^{0}$ contours on the source surface. In Fig. 6 we show contours of the magnitude of the magnetic field at 1 AU. Next, we consider a toroidal cloud propagating in the above IMF by applying the expansion rate mentioned in Sect. 2.4, viz. $r_{0}, R_{0} \propto r^{2 / 3}$, and solve Eqs. (4)-(6) numerically. The ratio of the internal to the external density was set to 0.5 . The aspect ratio was set equal to 2 and is constant during the entire evolution of the toroidal magnetic cloud. Also $r_{0}=0.02 \mathrm{AU}$ and $R_{0}=0.04 \mathrm{AU}$ at $r=$ 1 AU. This is a small, compact sample cloud comparable with the one interpreted in Romashets \& Vandas (2003).

The resulting velocity profiles of the toroidal cloud are shown in the Figs. 7 and 8. Figure 7 contains the profile of the radial velocity component while Fig. 8 contains the $\theta$-component and the $\varphi$-component profiles. For this case, the cloud was sume everywhere $g_{j}^{m}\left(t-r / V_{\mathrm{sw}}\right)$ and $h_{j}^{m}\left(t-r / V_{\mathrm{sw}}\right)$. All formulas remain the same, except for Eqs. (42) and (46), which are

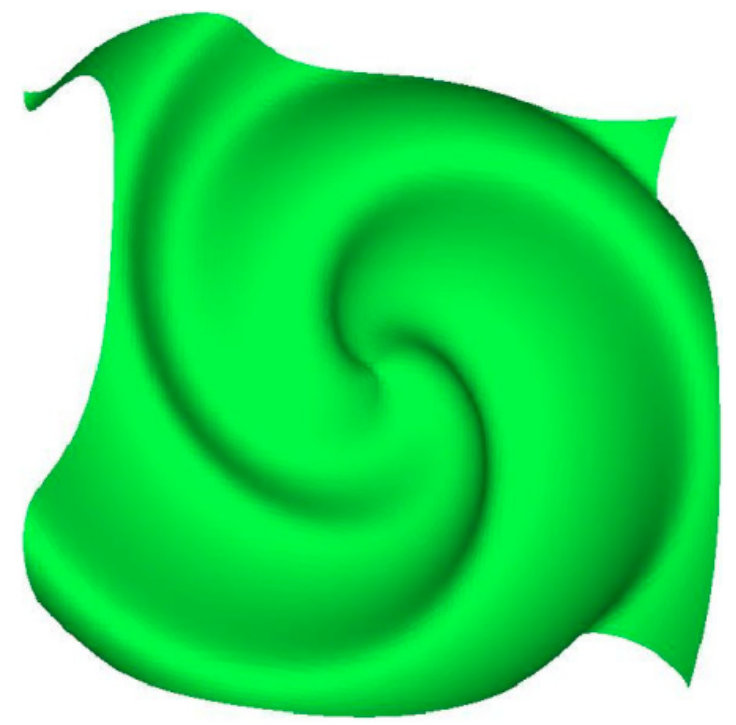

Fig. 2. Sector boundary of the interplanetary magnetic field (44)-(46). Below and above the surface $B_{r}$ has opposite signs.

The radial component decreases as $\frac{1}{r^{2}}$, the longitudinal component as $\frac{1}{r}$, and the $\theta$ component is derived from the solenoidal be equal to 1.1. It is set slightly different from unity in order to ensure $B_{\theta}=0$ at $r=r_{\mathrm{ss}}$ and since it is still close to unity the very similar to the Archimedean (or Parker) spiral. Solar rotation frequency is $\omega_{0}=\frac{2 \pi}{T_{0}}$, where $T_{0} \cong 27.5$ days. Here, $\varphi_{0}$ is angle for which the Sun rotates before the solar wind wind speed, usually $\varphi_{\mathrm{sw}} \simeq \frac{\pi}{4}$. One can see that $B_{r}$ is decreas $1 / r^{2}$, while $B_{\varphi}$ is decreasing as $1 / r$, according to Parker's model (1959). In turn $B_{\theta}$ remains comparatively small in the entire volme. At $1 \mathrm{AU}$ near the ecliptic plane, $B$ is of the order of 5 To satisfy the consistency we should have the maximum $B_{r}^{0}$ of

out $5 \times 10^{4} \mathrm{nT}$, which is comparable with photospheric valu The same approach works also when the harmonic Theoretically the sets can be updated every hour, using, 


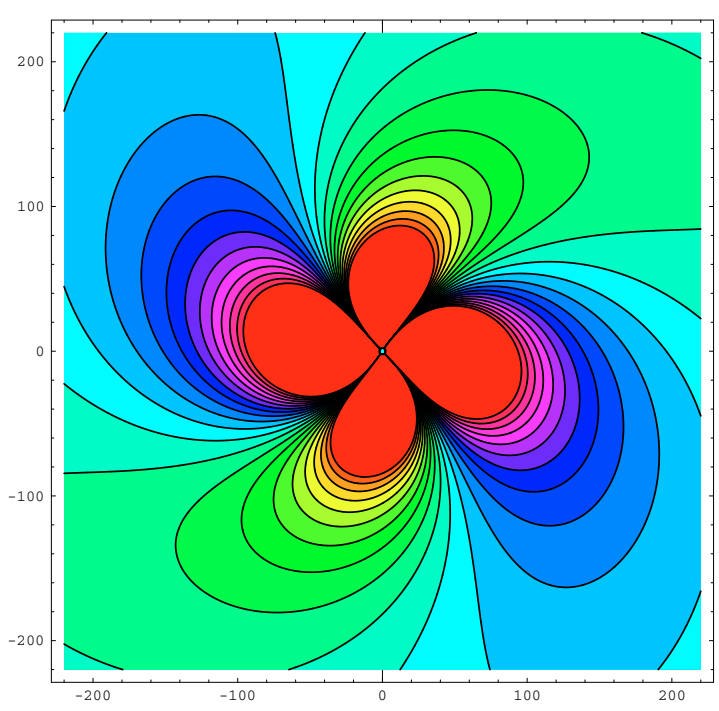

Fig. 3. Contours of $B_{r}$ in the plane $z=0$.

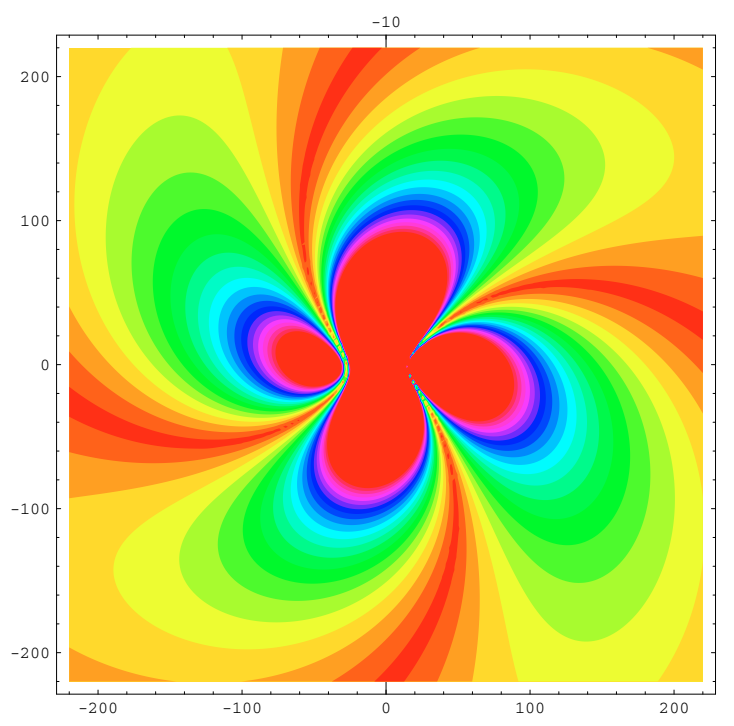

Fig. 4. Contours of $B$ in the plane $z=-10 R_{\mathrm{s}}$.

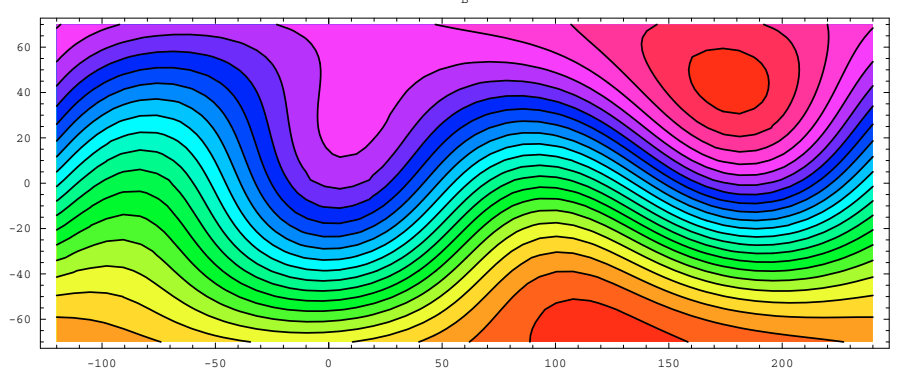

Fig. 5. Source-surface magnetic field, $B_{r}^{0}, r$-component at $r=2.5 R_{\mathrm{s}}$.

launched at $3 r_{\mathrm{s}}$ and $\theta_{0}=20^{\circ}$ and $\varphi_{0}=0^{\circ}$. At $1 \mathrm{AU}$, the cloud is at $\theta_{1}=10^{\circ}$ and $\varphi_{1}=-15^{\circ}$. The initial speed was $300 \mathrm{~km} \mathrm{~s}^{-1}$. This value is in accordance with results of Watari et al. 2005.

The cloud reached its maximal radial velocity of $1400 \mathrm{~km} \mathrm{~s}^{-1}$ at $10 R_{s}$ (see Fig. 7). The $v_{\varphi}$-component has a maximum of the order of $170 \mathrm{~km} \mathrm{~s}^{-1}$, and the maximum of $v_{\theta}$ is about $100 \mathrm{~km} \mathrm{~s}^{-1}$, or about $10 \%$ of the maximal radial velocity (see Fig. 8). The model background solar wind speed, shown in Fig. 7, is of the Parker (1959) type, radial, with its

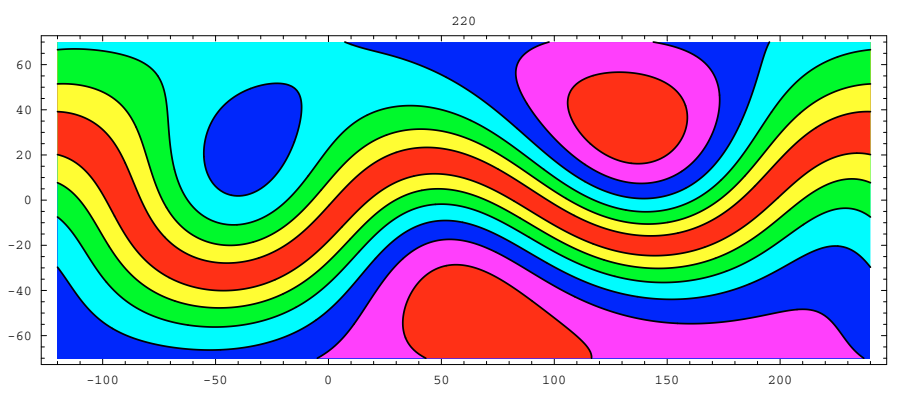

Fig. 6. Contours of $B$ on the sphere $r=220 R_{\mathrm{s}} \simeq r_{\mathrm{E}}$.

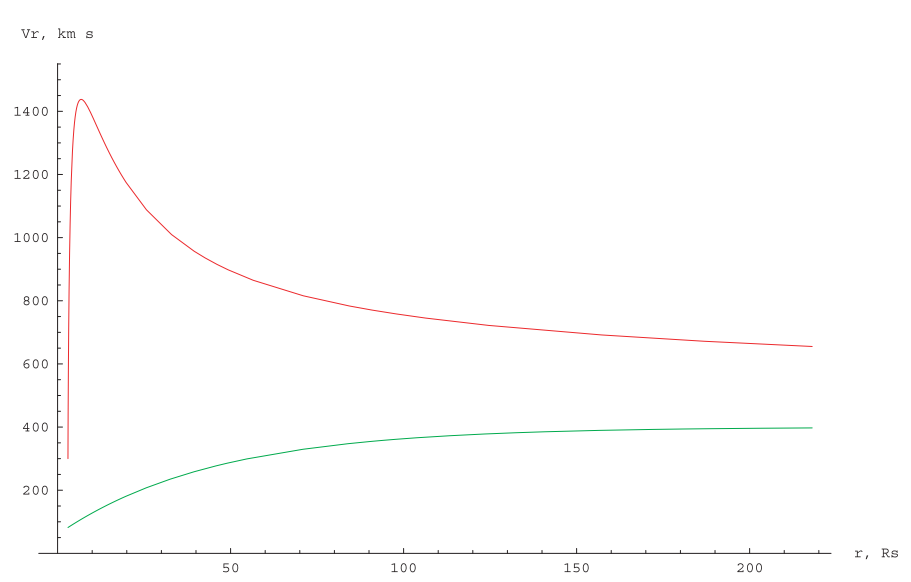

Fig. 7. Radial component of the velocity of the toroidal magnetic cloud (red). The model ambient solar wind speed is shown in green.

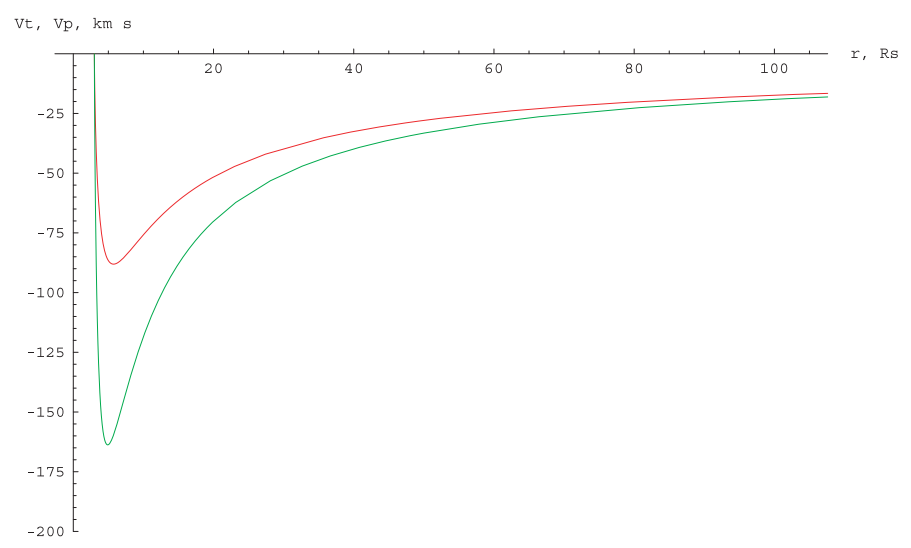

Fig. 8. The $\theta$-component of the velocity (in red) and the $\varphi$-component (in green).

maximum, $400 \mathrm{~km} \mathrm{~s}^{-1}$, at $r \rightarrow \infty$. In Fig. 9 the angular displacement of the cloud is shown. The result shows that small non-radial components are possible, and the cloud can be shifted during its propagation by 10-20 degrees. This problem was discussed by Webb et al. (2000) in their analysis of the May 14-15, 1997 event. The cloud came to the Earth's orbit below the ecliptic plane, while its most probable solar source was in the northern hemisphere. The derived velocity profiles (Fig. 7) are similar to those given by observations (Sheeley et al. 1999). In Figs. 7-9 the location and the velocity of the center of the cloud is shown while its distant parts, especially if the cloud expanded very rapidly, can be located even in the region $\pm 30^{\circ}$ from the center, for huge clouds. 


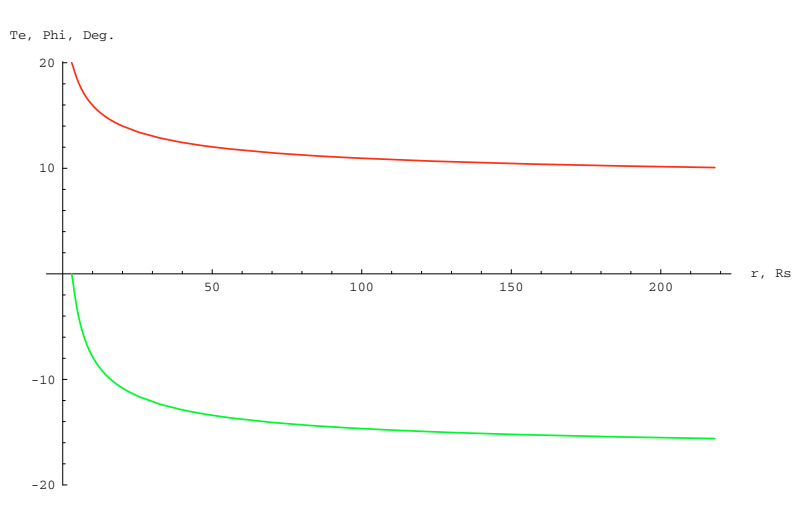

Fig. 9. Angular displacement during the propagation of the cloud: along $\theta$ (red curve) and along $\varphi$ (green curve).

\section{Perspective}

Similarly to the equation of motion, one can write an equation describing the rotation of the toroid using formulas for the moments of the diamagnetic force from Vandas et al. (2003). Let us define a differentially small angle of rotation around the $x$-axis by $\delta_{\tilde{x}}$ and around the $y$-axis by $\delta_{\tilde{y}}$. The corresponding angular velocities are $\omega_{\tilde{x}}=\dot{\delta}_{\tilde{x}}$ and $\omega_{y}=\dot{\delta}_{\tilde{y}}$, respectively. So we have the following relationships

$J \dot{\omega}_{\tilde{x}}=J \ddot{\delta}_{\tilde{x}}=M_{\tilde{x}}$,

$J \dot{\omega}_{\tilde{y}}=J \ddot{\delta}_{\tilde{y}}=M_{\tilde{x}}$

Here, $J=1 / 2 M_{c}\left(R_{0}^{2}+5 / 4 r_{0}^{2}\right)$ is the toroid's moment of inertia, and $M_{\tilde{x}}$ and $M_{\tilde{y}}$ are components of the the moment of the diamagnetic force:

$\boldsymbol{M}=-\frac{1}{2 \tilde{\mu}_{0}} \oint\left(B_{\eta}^{2}+B_{\varphi}^{2}\right)(\boldsymbol{r} \times \mathrm{d} \boldsymbol{S})$,

which can be expressed in the toroid's (tilde) system as:

$$
\begin{aligned}
& M_{\tilde{x}}=-\frac{1}{2 \tilde{\mu}_{0}} \int_{-\pi}^{\pi} \int_{0}^{2 \pi}\left(B_{\eta}^{2}+B_{\varphi}^{2}\right)\left(\tilde{y} w_{\tilde{z}}-\tilde{z} w_{\tilde{y}}\right) \mathrm{d} \varphi \mathrm{d} \eta, \\
& M_{\tilde{y}}=-\frac{1}{2 \tilde{\mu}_{0}} \int_{-\pi}^{\pi} \int_{0}^{2 \pi}\left(B_{\eta}^{2}+B_{\varphi}^{2}\right)\left(\tilde{z} w_{\tilde{x}}-\tilde{x} w_{\tilde{z}}\right) \mathrm{d} \varphi \mathrm{d} \eta .
\end{aligned}
$$

Remark that $M_{\tilde{z}}=0$ because of symmetry, so that we have considered here only two components of the moment.

One can integrate Eq. (48) numerically or analytically. A simple Euler scheme, e.g. would read as follows:

$\omega_{\tilde{x}}^{k+1}=\frac{M_{\tilde{x}}}{J} \Delta t+\omega_{\tilde{x}}^{k}, \quad \delta_{\tilde{x}}^{k+1}=\omega_{\tilde{x}}^{k+1} \Delta t$,

$\omega_{\tilde{y}}^{k+1}=\frac{M_{\tilde{y}}}{J} \Delta t+\omega_{\tilde{y}}^{k}, \quad \delta_{\tilde{y}}^{k+1}=\omega_{\tilde{y}}^{k+1} \Delta t$.

The value of the angular velocity from the previous step (at $t_{k}$ ) is denoted as $\omega^{k}$. The value of $\Delta t$ must be taken small enough. In order to make the following step it is necessary to change the local system, i.e. to rotate the cloud. The relationship between the vectors $\boldsymbol{a}$ at time steps $k$ and $k+1$, in the local coordinate system of the toroid is given by:

$\boldsymbol{a}_{T}^{k+1}=A_{k} \cdot \boldsymbol{a}_{T}^{k}$.
The matrix of $x y$ rotation (around the $x$-axis by $\delta_{\tilde{x}}$ and around the $y$-axis by $\left.\delta_{\tilde{y}}\right), A_{k}$, is given by:

$\left(\begin{array}{lll}\cos \delta_{\tilde{y}}^{k+1} & 0 & \sin \delta_{\tilde{y}}^{k+1} \\ \sin \delta_{\tilde{x}}^{k+1} \sin \delta_{\tilde{y}}^{k+1} & \cos \delta_{\tilde{x}}^{k+1} & -\sin \delta_{\tilde{x}}^{k+1} \cos \delta_{\tilde{y}}^{k+1} \\ -\cos \delta_{\tilde{x}}^{k+1} \sin \delta_{\tilde{y}}^{k+1} & \sin \delta_{\tilde{x}}^{k+1} & \cos \delta_{\tilde{x}}^{k+1} \cos \delta_{\tilde{y}}^{k+1}\end{array}\right)$.

One can see that $\boldsymbol{a}_{T}^{k}$ was $(0,0,1)$ and $\boldsymbol{a}_{T}^{k+1}$ is $\left(\sin \delta_{\tilde{y}}^{k+1},-\sin \delta_{\tilde{x}}^{k+1} \cos \delta_{\tilde{y}}^{k+1}, \quad \cos \delta_{\tilde{x}}^{k+1} \cos \delta_{\tilde{y}}^{k+1}\right)$. To find the vector $\boldsymbol{a}$ in SEC one should apply a reverse transformation matrix $W_{k+1}$ :

$\left(\begin{array}{lll}x_{1} & y_{1} & z_{1} \\ x_{2} & y_{2} & z_{2} \\ x_{3} & y_{3} & z_{3}\end{array}\right)$

Here, $x_{1}, x_{2}$, and $x_{3}$ are components of the vector $x$ defined by Eq. (1), in SEC, etc. Then

$\boldsymbol{a}^{k+1}=W_{k+1} \cdot \boldsymbol{a}_{T}^{k+1}$.

Having from the equation of motion also $\boldsymbol{r}^{k+1}$, we can return to the set of Eqs. (1)-(3) and repeat the calculation for $k+2$ etc.

\section{Conclusions}

A new approach for the study of the dynamics of interplanetary magnetic clouds in a three-dimensional time-dependent solar wind has been proposed and demonstrated. An analytical description of the 3D heliospheric ballerina skirt type field, consistent with solar magnetic observations, and with in-situ measurements at $1 \mathrm{AU}$ was derived and illustrated and used for some sample calculations. The three dimensional motion and rotation of a toroidally shaped CME/magnetic cloud through this model field was determined for typical conditions in the solar wind. The obtained velocity profiles are consistent with those determined earlier from a data analysis (Sheeley et al. 1999) for typical CMEs. The latitudinal and longitudinal motion of a CME on its way from the Sun to the Earth's orbit, was modeled by Vandas et al. (1996) and demonstrated in a numerical MHD simulation by the same authors. The three-dimensional velocity profiles and trajectories calculated for sample clouds show that slight non-radial velocity components can occur during the cloud's motion to the Earth's orbit, and the angular displacement can easily reach values of 20 degrees. To study more deeply the dynamics of the clouds in the 3D nonstationary interplanetary medium, one should use the newly developed MHD codes (Poedts et al. 2005; Odstrcil et al. 2005; Chané et al. 2005; Chané et al. 2006; van der Holst et al. 2005), taking into account general features of this motion, which can be determined from analytical considerations, such as the one presented in this paper.

Acknowledgements. The authors thank numerous colleagues for their encouragement of our work on solar wind models. Specifically helpful comments were made by Murray Dryer. The work was supported by BELSPO, RFBR grant 0605-64500, EU/INTAS grant 03-51-6206, AV ČR project 1QS300120506, and GA ČR grant 205/06/0875. These results were obtained in the framework of the projects GOA 2004/01 (K.U.Leuven), G.0304.07 (FWO-Vlaanderen) and C90203 (ESA Prodex 8).

\section{Appendix A: Coefficients $C_{i}$}

The coefficients $C_{i}$ in Eq. (15) are given by

$C_{1}=B_{0 x} \sin \alpha-B_{0 y} \cos \alpha \cos \beta-B_{0 z} \cos \alpha \sin \beta$,

$C_{2}=B_{0 y} \sin \beta-B_{0 z} \cos \beta$, 


$$
\begin{aligned}
C_{3}= & B_{0 x} \cos \alpha+B_{0 y} \sin \alpha \cos \beta+B_{0 z} \sin \alpha \sin \beta, \\
C_{4}= & \frac{1}{4 L}\left[B_{1 x x}\left(\sin ^{2} \alpha-\cos ^{2} \alpha \sin ^{2} \beta+\cos ^{2} \beta\right)\right. \\
& -B_{1 x y} \sin 2 \alpha \cos \beta+B_{1 y y}\left(1+\cos ^{2} \alpha\right) \cos 2 \beta \\
& \left.-B_{1 x z} \sin 2 \alpha \sin \beta+B_{1 y z}\left(1+\cos ^{2} \alpha\right) \sin 2 \beta\right], \\
C_{5}= & \frac{1}{2 L}\left[B_{1 x x}\left(\sin ^{2} \alpha \sin ^{2} \beta-\cos ^{2} \alpha\right)\right. \\
& -B_{1 x y} \sin 2 \alpha \cos \beta-B_{1 y y} \sin ^{2} \alpha \cos 2 \beta \\
& \left.-B_{1 x z} \sin 2 \alpha \sin \beta-B_{1 y z} \sin ^{2} \alpha \sin 2 \beta\right], \\
C_{6}= & \frac{1}{L}\left(-B_{1 x x} \cos \alpha \cos \beta \sin \beta+B_{1 x y} \sin \alpha \sin \beta\right. \\
& -B_{1 x z} \sin \alpha \cos \beta-B_{1 y y} \cos \alpha \sin 2 \beta \\
& \left.+B_{1 y z} \cos \alpha \cos 2 \beta\right), \\
C_{7}= & \frac{1}{L}\left[B_{1 x x} \cos \alpha \sin \alpha\left(1+\sin ^{2} \beta\right)\right. \\
& -B_{1 x y} \cos 2 \alpha \cos \beta-B_{1 x z} \cos 2 \alpha \sin \beta \\
& \left.-B_{1 y y} \cos \alpha \sin \alpha \cos 2 \beta-B_{1 y z} \sin 2 \alpha \sin \beta \cos \beta\right], \\
C_{8}= & \frac{1}{L}\left(B_{1 x x} \sin \alpha \cos \beta \sin \beta\right. \\
& +B_{1 x y} \cos \alpha \sin \beta-B_{1 x z} \cos \alpha \cos \beta \\
& \left.+B_{1 y y} \sin \alpha \sin 2 \beta-B_{1 y z} \sin \alpha \cos 2 \beta\right) . \\
&
\end{aligned}
$$

\section{Appendix B: Harmonic functions $\Phi_{0}^{i}$}

The harmonic functions $\Phi_{0}^{i}$ in Eq. (16) can be expressed as a sum of toroidal harmonics:

$$
\begin{aligned}
\Phi_{0}^{1}= & \sqrt{\cosh \mu-\cos \eta} \frac{2^{\frac{3}{2}} a}{\pi} \\
& \times \sum_{n=-\infty}^{\infty} Q_{n-\frac{1}{2}}^{1}(\cosh \mu) \cos n \eta \cos \varphi \\
\Phi_{0}^{2}= & \sqrt{\cosh \mu-\cos \eta} \frac{2^{\frac{3}{2}} a}{\pi} \\
& \times \sum_{n=-\infty}^{\infty} Q_{n-\frac{1}{2}}^{1}(\cosh \mu) \cos n \eta \sin \varphi, \\
\Phi_{0}^{3}= & \sqrt{\cosh \mu-\cos \eta} \frac{2^{\frac{3}{2}} a}{\pi} \\
& \times \sum_{n=-\infty}^{\infty} Q_{n-\frac{1}{2}}(\cosh \mu) n \sin n \eta, \\
\Phi_{0}^{4}= & \sqrt{\cosh \mu-\cos \eta} \frac{2^{\frac{5}{2}} a^{2}}{3 \pi} \\
& \times \sum_{n=-\infty}^{\infty} Q_{n-\frac{1}{2}}^{2}(\cosh \mu) \cos n \eta \cos 2 \varphi, \\
\Phi_{0}^{6}= & \sqrt{\cosh \mu-\cos \eta} \frac{2^{\frac{3}{2}} a^{2}}{3 \pi} \\
& \times \sum_{n=-\infty}^{\infty} Q_{n-\frac{1}{2}}^{2}(\cosh \mu) \cos n \eta \sin 2 \varphi, \\
\Phi_{0}^{5}= & \sqrt{\cosh \mu-\cos \eta} \frac{2^{\frac{3}{2}} a^{2}}{\pi} \\
& \times \sum_{n=-\infty}^{\infty} Q_{n-\frac{1}{2}}(\cosh \mu)\left(\frac{1}{4}+n^{2}\right) \cos n \eta, \\
& \\
& \\
& \\
&
\end{aligned}
$$

$$
\begin{aligned}
\Phi_{0}^{7}= & \sqrt{\cosh \mu-\cos \eta} \frac{2^{\frac{5}{2}} a^{2}}{3 \pi} \\
& \times \sum_{n=-\infty}^{\infty} Q_{n-\frac{1}{2}}^{1}(\cosh \mu) n \sin n \eta \cos \varphi, \\
\Phi_{0}^{8}= & \sqrt{\cosh \mu-\cos \eta} \frac{2^{\frac{5}{2}} a^{2}}{3 \pi} \\
& \times \sum_{n=-\infty}^{\infty} Q_{n-\frac{1}{2}}^{1}(\cosh \mu) n \sin n \eta \sin \varphi .
\end{aligned}
$$

$Q_{n-\frac{1}{2}}^{m}$ denote the Legendre functions of the second kind (vanishing in the central line of the toroid). A method how to obtain these relationships is described in Romashets \& Vandas (2001).

\section{Appendix C: Coefficients $K_{i}$ and functions $N_{i}, H_{n}^{i}$, and $G_{n}^{i}$}

The coefficients $K_{i}$ and functions $N_{i}, H_{n}^{i}$, and $G_{n}^{i}$ are:

$$
\begin{aligned}
& K_{1}=\frac{2^{3 / 2} a}{\pi}, K_{2}=K_{1}, K_{3}=K_{1}, \\
& K_{4}=\frac{2^{5 / 2} a^{2}}{3 \pi}, K_{5}=\frac{2^{3 / 2} a^{2}}{\pi},
\end{aligned}
$$$$
K_{6}=\frac{2^{3 / 2} a^{2}}{3 \pi}, K_{7}=K_{4}, K_{8}=K_{4},
$$

$G_{n}^{1}(x)=Q_{n-\frac{1}{2}}^{1}(x)-a_{n}^{1} P_{n-\frac{1}{2}}^{1}(x)$,

$G_{n}^{2}(x)=Q_{n-\frac{1}{2}}^{1}(x)-a_{n}^{2} P_{n-\frac{1}{2}}^{1}(x)$,

$G_{n}^{3}(x)=Q_{n-\frac{1}{2}}(x)-a_{n}^{3} P_{n-\frac{1}{2}}(x)$,

$G_{n}^{4}(x)=Q_{n-\frac{1}{2}}^{2}(x)-a_{n}^{4} P_{n-\frac{1}{2}}^{2}(x)$,

$G_{n}^{5}(x)=Q_{n-\frac{1}{2}}(x)-a_{n}^{5} P_{n-\frac{1}{2}}(x)$,

$G_{n}^{6}(x)=Q_{n-\frac{1}{2}}^{2}(x)-a_{n}^{6} P_{n-\frac{1}{2}}^{2}(x)$,

$G_{n}^{7}(x)=Q_{n-\frac{1}{2}}^{1}(x)-a_{n}^{7} P_{n-\frac{1}{2}}^{1}(x)$,

$G_{n}^{8}(x)=Q_{n-\frac{1}{2}}^{1}(x)-a_{n}^{8} P_{n-\frac{1}{2}}^{1}(x)$,

$H_{n}^{1}(\eta)=\cos n \eta, H_{n}^{2}(\eta)=H_{n}^{1}(\eta), H_{n}^{3}(\eta)=n \sin n \eta$,

$H_{n}^{4}(\eta)=H_{n}^{1}(\eta), H_{n}^{5}(\eta)=\left(n^{2}+\frac{1}{4}\right) H_{n}^{1}(\eta)$,

$H_{n}^{6}(\eta)=H_{n}^{1}(\eta), H_{n}^{7}(\eta)=H_{n}^{3}(\eta), H_{n}^{8}(\eta)=H_{n}^{3}(\eta)$,

$N_{1}(\varphi)=\cos \varphi, N_{2}(\varphi)=\sin \varphi, N_{3}(\varphi)=1$,

$N_{4}(\varphi)=\cos 2 \varphi, N_{5}(\varphi)=N_{3}(\varphi), N_{6}(\varphi)=\sin 2 \varphi$,

$N_{7}(\varphi)=N_{1}(\varphi), N_{8}(\varphi)=N_{2}(\varphi)$.

Notice that, obviously,

$G_{n}^{i}(x)=G_{-n}^{i}(x) \quad$ and $\quad H_{n}^{i}(\eta)=H_{-n}^{i}(\eta)$ 


\section{Appendix D: Calculation of the coefficients $a_{n}^{i}$}

For $i=1$, condition 1 can be symbolically expressed from Eq. (23) as

$\sum_{n=-\infty}^{\infty}\left[\left(R_{n}^{1}-S_{n}^{1} a_{n}^{1}\right)+2\left(T_{n}^{1}-U_{n}^{1} a_{n}^{1}\right) \cos \eta\right] \cos n \eta=0$,

where

$R_{n}^{1}=Q_{n-\frac{1}{2}}^{1}\left(\cosh \mu_{0}\right)+2 \cosh \mu_{0} Q_{n-\frac{1}{2}}^{1 \prime}\left(\cosh \mu_{0}\right)$,

$S_{n}^{1}=P_{n-\frac{1}{2}}^{1}\left(\cosh \mu_{0}\right)+2 \cosh \mu_{0} P_{n-\frac{1}{2}}^{1 \prime}\left(\cosh \mu_{0}\right)$,

$T_{n}^{1}=-Q_{n-\frac{1}{2}}^{1 \prime}\left(\cosh \mu_{0}\right)$,

$U_{n}^{1}=-P_{n-\frac{1}{2}}^{1 \prime}\left(\cosh \mu_{0}\right)$.

With the use of the relationship

$2 \cos n \eta \cos \eta=\cos (n-1) \eta+\cos (n+1) \eta$,

one can express Eq. (D.1) as a sum of terms with $\cos n \eta$,

$$
\begin{aligned}
& \sum_{n=-\infty}^{\infty}\left(R_{n}^{1}-S_{n}^{1} a_{n}^{1}+T_{n-1}^{1}-U_{n-1}^{1} a_{n-1}^{1}+T_{n+1}^{1}\right. \\
& \left.-U_{n+1}^{1} a_{n+1}^{1}\right) \cos n \eta=0,
\end{aligned}
$$

and equating the coefficients in this equation to zero yields the infinite system of linear equations for the unknowns $a_{n}^{1}$

$$
U_{n-1}^{1} a_{n-1}^{1}+S_{n}^{1} a_{n}^{1}+U_{n+1}^{1} a_{n+1}^{1}=T_{n-1}^{1}+R_{n}^{1}+T_{n+1}^{1} .
$$

The coefficients $a_{n}^{1}$ are decreasing rapidly with $n$. For numerical purposes we restricted ourselves to $|n| \leq 10$ and we solved the resulting closed system (D.8) of 11 equations. The system (D.8) is almost the same for all $i$, only the following changes in (D.2)-(D.5) must be made: for $i=2$ : no changes;

$$
\begin{aligned}
& \text { for } i=3: Q_{n-\frac{1}{2}}^{1} \rightarrow n Q_{n-\frac{1}{2}} \text { and } P_{n-\frac{1}{2}}^{1} \rightarrow n P_{n-\frac{1}{2}} \\
& \text { for } i=4: Q_{n-\frac{1}{2}}^{1} \rightarrow Q_{n-\frac{1}{2}}^{2} \text { and } P_{n-\frac{1}{2}}^{1} \rightarrow P_{n-\frac{1}{2}}^{2} ; \\
& \text { for } i=5: Q_{n-\frac{1}{2}}^{1} \rightarrow\left(\frac{1}{4}+n^{2}\right) Q_{n-\frac{1}{2}} \text { and }
\end{aligned}
$$

$$
\begin{aligned}
& P_{n-\frac{1}{2}}^{1} \rightarrow\left(\frac{1}{4}+n^{2}\right) P_{n-\frac{1}{2}} ; \\
& \text { for } i=6: Q_{n-\frac{1}{2}}^{1} \rightarrow Q_{n-\frac{1}{2}}^{2} \text { and } P_{n-\frac{1}{2}}^{1} \rightarrow P_{n-\frac{1}{2}}^{2} \\
& \text { for } i=7: Q_{n-\frac{1}{2}}^{1} \rightarrow n Q_{n-\frac{1}{2}}^{1} \text { and } P_{n-\frac{1}{2}}^{1} \rightarrow n P_{n-\frac{1}{2}}^{1} \\
& \text { for } i=8: Q_{n-\frac{1}{2}}^{1} \rightarrow n Q_{n-\frac{1}{2}}^{1} \text { and } P_{n-\frac{1}{2}}^{1} \rightarrow n P_{n-\frac{1}{2}}^{1}
\end{aligned}
$$

\section{References}

Cane, H. V., Kahler, S. W., \& Sheeley, N. R. Jr. 1986, JGR, 91, 13321

Cargill, P. J., Chen, J., Spicer, D. S., \& Zalesak, S. T. 1996, JGR, 101, 4855

Chandrasekhar, S., \& Kendall, P. C. 1957 ApJ, 126, 457

Chané, E., Jacobs, C., Van der Holst, B., Poedts, S., \& Kimpe, D. 2005, A\&A, 432, 331-339

Chane, E., van der Holst, B., Jacobs, C., Poedts, S., \& Kimpe, D. 2006, A\&A, 447,727

Howard, T. A., Webb, D. F., Tappin, S. J., Mizuno, D. R., \& Johnston, J. C. 2006, JGR, 111

Ivanov, K. G., Kharshiladze, A. F., \& Romashets, E. P. 1992, GA, 32, 25

Ivanov K. G., Styazkin V. A., Eroshenko E. G., \& Romashets, E. P. 1996, in Proc. of Solar Wind Eight, edited by D. Winterhalter et al., AIP Conf. Proc., 382,575

Kotova, G. A., Gringauz, K. I., Bezrukikh, V. V., Verigin, M. I., \& Shvachunova, L. A. 1987, CR, 25, 93

Parker, E. N. 1957, ApJS, 3, 51

Parker, E. N. 1959, JGR, 64, 1675

Odstrcil, D., Pizzo, V. J., \& Arge, C. N. 2005, JGR, 110

Pneuman, G. W. 1984, SP 94, 387

Poedts, S., Chané, E., van der Holst, B., et al. 2005, in Proc. Solar Wind 11 / SOHO 16, ed. B. Fleck et al., ESA, SP-592, 301

Reiner, M. J., Kaiser, M. L., \& Bougeret, J.-L. 2005, in Proceedings of the Solar Wind 11 / SOHO 16, ed. B. Fleck et al., ESA SP-592, 307

Romashets, E. P., \& Vandas, M. 2001, JGR, 10610615

Romashets, E. P., \& Vandas, M. 2003, GRL, 30, 2065

Romashets, E. P., \& Vandas, M. 2005a, in Proc. IAU Symp. 226, ed. K. Dere et al., 428

Romashets, E., \& Vandas, M. 2005b, in Proc. Solar Wind 11 / SOHO 16, ed. B. Fleck et al., ESA SP-592, 156

Sheeley, N. R., Walters, J. H., Wang, Y.-M., \& Howard, R. A. 1999, JGR, 104, 24739

Vandas M., Fischer S., Dryer M., Smith Z., \& Detman T. 1996, JGR, 101, 2505

Vandas, M., Romashets, E. P., \& Watari, S. 2003, A\&A, 412, 281

van der Holst, B., Poedts, S., Chané, E., et al. 2005, SSR, 121, 91

Watari, S., Vandas, M., \& Watanabe, T. 2005, in Proceedings of the IAU Symposium 226, editors K. Dere et al., 433

Webb, D. F., Lepping, R. P., Burlaga, L. F., et al. 2000, JGR, 105, 27251

Winterhalter, D., Smith, E. J., Burton, M. E., Murphy, N., \& McComas, D. J. 1994, JGR, 99, 6667 